\title{
Age estimation by multidetector CT images of the sagittal suture
}

\author{
Fumiko Chiba • Yohsuke Makino • Ayumi Motomura • \\ Go Inokuchi • Suguru Torimitsu • Namiko Ishii • Ayaka Sakuma • \\ Sayaka Nagasawa • Hisako Saitoh • Daisuke Yajima • \\ Mutsumi Hayakawa $\cdot$ Yuriko Odo $\cdot$ Yoichi Suzuki $\cdot$ Hirotaro Iwase
}

Received: 20 December 2012 / Accepted: 27 May 2013 / Published online: 13 June 2013

(C) The Author(s) 2013. This article is published with open access at Springerlink.com

\begin{abstract}
Closure of cranial sutures progresses with age; therefore, macroscopic assessment of cranial sutures has been used as one method of age estimation. Postmortem computed tomography (PMCT), which many forensic medical departments and institutes have begun to adopt, has the potential to simplify the gathering of information from cranial sutures for both surface and cross-sectional evaluation. To examine the feasibility of age estimation by cross-sectional multidetector computed tomography images of the sagittal suture, PMCT findings of 125 subjects of known age and sex were retrospectively reviewed. The sagittal suture was divided into four segments, and 20 cross-sectional slices from each segment were analyzed. These slices were each categorized by visual evaluation into one of the seven stages defined by Harth et al.
\end{abstract}

F. Chiba $(\bowtie) \cdot$ Y. Makino $\cdot$ A. Motomura $\cdot$ G. Inokuchi

S. Torimitsu $\cdot$ N. Ishii $\cdot$ A. Sakuma $\cdot$ S. Nagasawa $\cdot$ H. Saitoh $\cdot$

D. Yajima $\cdot$ M. Hayakawa $\cdot$ Y. Odo $\cdot$ H. Iwase

Department of Legal Medicine, Graduate School of Medicine,

Chiba University, Inohana 1-8-1, Chuo-ku,

Chiba City, Chiba Prefecture 260-8670, Japan

e-mail: chibafumico@chiba-u.jp

Y. Makino

Department of Radiology, Graduate School of Medicine, Chiba

University, Inohana 1-8-1, Chuo-ku,

Chiba City, Chiba Prefecture 260-8670, Japan

Y. Suzuki

Department of Public Health, Graduate School of Medicine, Chiba University, Inohana 1-8-1, Chuo-ku,

Chiba City, Chiba Prefecture 260-8670, Japan

Y. Suzuki

Department of Education and Training, Tohoku Medical Megabank Organization, Tohoku University, 2-1 Seiryo-machi, Aoba-ku, Sendai 980-8573, Japan according to the degree of closure. The mean stage value of 20 slices was calculated for each segment. We were able to evaluate cross-sectional images of the sagittal suture by PMCT, and a positive correlation between age and closure degree was observed. Despite the prediction interval achieved with this method not being superior to traditional macroscopic or flat-panel CT assessment, multidetector CT is a potentially useful tool, in conjunction with other methods, for age estimation, particularly in adult females and in cases where only a skull is the sole remain.

Keywords Age determination - Forensic anthropology · Multidetector computed tomography $\cdot$ Cranial suture

\section{Introduction}

Age estimation is one of the most important methods used in the identification of human remains and is also one of the greatest challenges in forensic medicine particularly in the case of adult remains. Over the last few years, the use of imaging modalities such as computed tomography (CT), magnetic resonance imaging, and ultrasonography in age estimation has been investigated alongside the reevaluation of classic techniques such as estimation based on cranial sutures [1-13]. Cranial suture closure progresses with age, and therefore, the macroscopic assessment of the surface of cranial sutures has long been used as a method of age estimation [14-17]. Some reports suggest that cranial suture-based age estimation should be avoided because the degree of closure of cranial sutures demonstrates large individual differences [8-20]. However, thus far, no definitive method of age estimation has been established, and cranial suture evaluation remains essential 
in this field of forensics, especially when the skull is the sole remain.

Traditionally, evaluation of cranial sutures involves assessment of ecto- and endocranial sutures with the naked eye, requiring the removal of skin, temporal muscles, and epicranial aponeurosis from cranial bones. Evaluation of the sagittal suture with modern radiographic modalities such as $\mathrm{CT}$ would enable assessment without removal of the soft tissue. Many forensic departments and institutes have begun to adopt postmortem CT (PMCT), and several reports in recent years have attempted to estimate age using PMCT, by examination of the pelvis, clavicle, premolar tooth, spheno-occipital synchondrosis, first rib, fourth rib, or thyroid cartilage [1-7].

Harth et al. scanned cranial bones with $\mathrm{CT}$ and assessed the cross section of sutures; however, this study was performed using flat-panel $\mathrm{CT}$ and cranial bones from which the soft tissue had been removed [8,9]. Flat-panel CT is superior to multidetector CT (MDCT) in terms of spatial resolution and is considered suitable for the evaluation of sutures. However, the lower dynamic range, scatter radiation, and cone beam artifact make it inferior in the imaging of soft tissue, making it unsuitable for systemic evaluation.

Postmortem CT is typically performed using MDCT in forensic departments and institutes equipped with CT. Thus, if the evaluation of sutures by MDCT without removing soft tissue is possible during routine PMCT systemic evaluation, it could contribute to a reduction in the time and cost of forensic investigations. Here, we performed a retrospective review to examine the feasibility of age estimation by evaluation of MDCT cross-sectional images of sagittal sutures. To the best of our knowledge, this study is the first to investigate the application of MDCT for age estimation by sagittal suture evaluation.

\section{Materials and methods}

This study reviewed data from 125 subjects of known age and sex who underwent PMCT with subsequent forensic autopsy at the Department of Legal Medicine at Chiba University between October 2009 and November 2011. Cases were excluded if the history highlighted conditions or events that could have affected the cranial sutures. For example, skull fracture, burning, cranial anomalies such as stenocephaly, childhood abuse, or diseases likely to have affected ossification were excluded. Subjects included 64 men (median age, 55 years; quartile deviation, 15.38 years) and 61 women (median age, 67 years; quartile deviation, 23.5 years).

Postmortem CT was performed with 16-row detector CT (Eclos, Hitachi Medical Corporation, Tokyo, Japan). The scanning protocol was as follows: collimation of $0.625 \mathrm{~mm}$, reconstruction interval of $0.63 \mathrm{~mm}$, tube voltage of $120 \mathrm{kV}$, tube current of $200 \mathrm{~mA}$, and rotation time of $1 \mathrm{r} / \mathrm{s}$. A hard filter was used. Image data were processed on a workstation (Synapse Vincent, Fujifilm Medical, Tokyo, Japan) to obtain orthogonal multiplanar reconstruction images and volume rendering technique images. Reconstructed cross-sectional images were viewed using a window width of $1,600 \mathrm{HU}$ and a window level of 1,000 HU as described previously [8].

The sagittal suture was chosen for evaluation owing to its position at approximately the midline of the skull, which means that the making of reconstruction images of this suture is relatively easier than that of other cranial sutures. For assessment, a three-dimensional image of the sagittal suture was divided into four segments, and a $10-\mathrm{mm}$ block was chosen from each segment (Fig. 1). A stack of 20 crosssectional images (i.e., perpendicular to the sagittal suture) of 0.5 -mm thickness was reconstructed from each block. These images were each categorized by visual evaluation into one of seven stages according to the degree of closure as defined by Harth et al. (Fig. 2; Table 1) [8]. All images were evaluated by a single assessor, and intra- and inter-observer reproducibility was tested by randomly selecting 30 subjects for reevaluation by both the original and a second assessor. For each segment, 20 stage classification scores were recorded from which three different representative scores were calculated. Firstly, in the "Frequency method," the representative score was the stage value with the highest occurrence rate in the 20 slices.

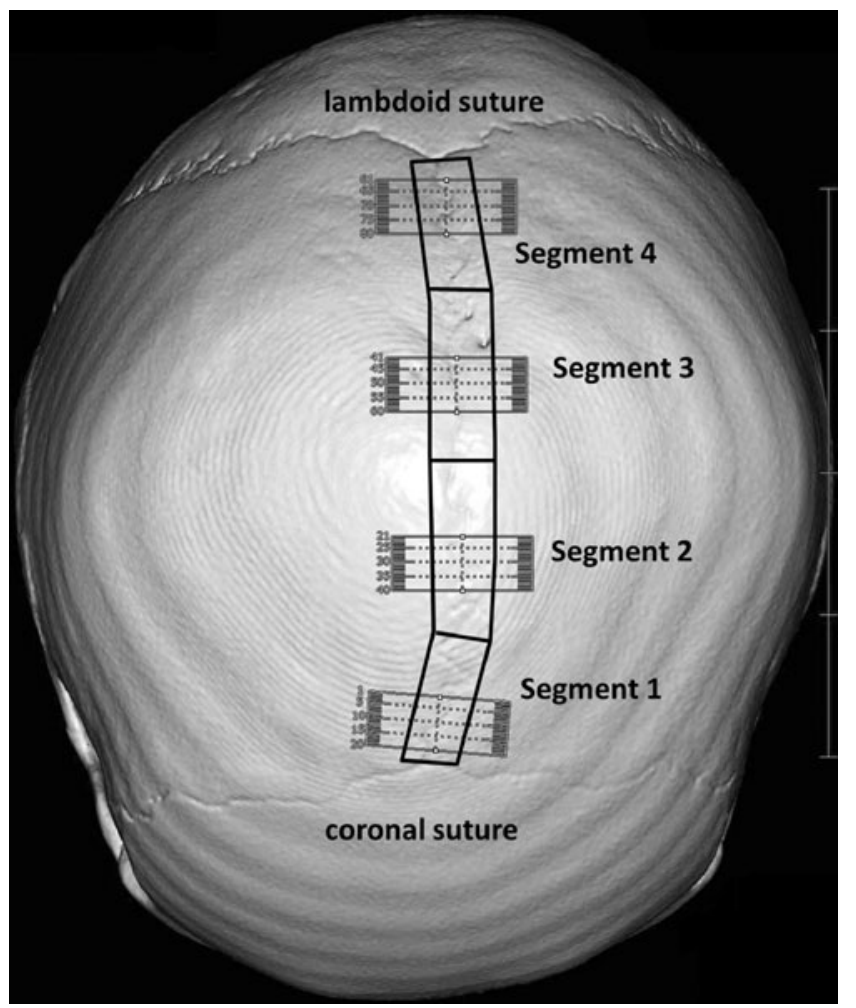

Fig. 1 Volume rendering image of the skull reconstructed by CT workstation. The sagittal suture was divided into four segments, and a 10-mm block was chosen from each segment for cross-sectional images 


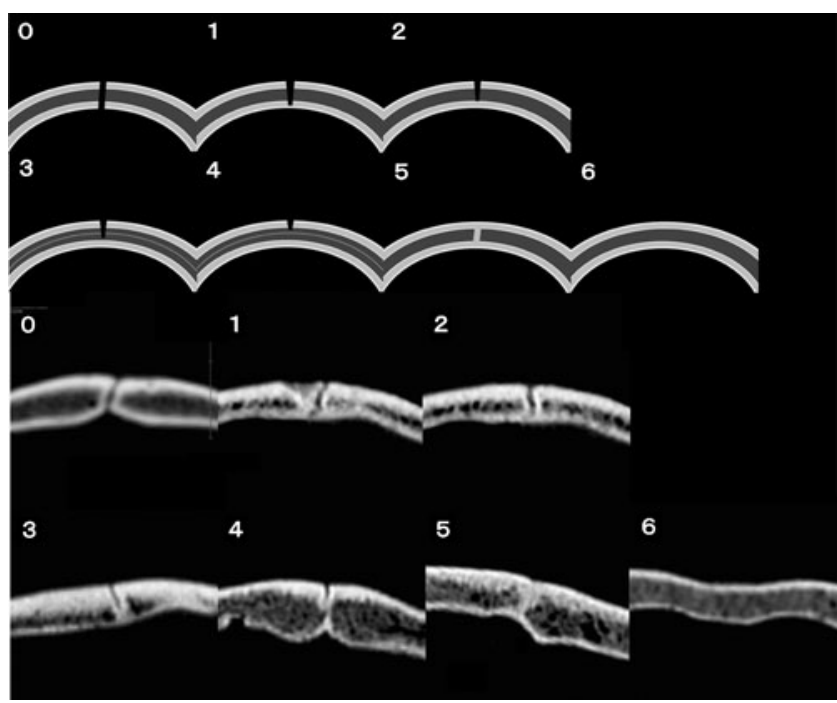

Fig. 2 Images of the seven stages of suture closure for MDCT evaluation. The upper two rows are a schematic diagram of the seven stages of closure, and the lower two rows are three-dimensional reconstruction images of the seven stages

Secondly, in the "Average method," the representative score was the mean stage value of the 20 slices. Finally, in the "Maximum method," the representative score was the highest stage value in the 20 slices. Each segment was assigned a score according to these three methods, and the mean score of the four segments was calculated as the representative score of the whole suture.

To investigate the degree of correlation between the age and the degree of closure as assessed by the three methods, the Pearson product-moment correlation coefficient was calculated. To determine the strongest correlation, the statistical significance of the Pearson product-moment correlation coefficient was compared across the three methods [21, 22]. To compare measures of central tendency, the $t$ test was used for normally distributed data, and the Mann-Whitney $U$ test and Wilcoxon test were used for non-normally distributed data. $P$ values less than 0.05 were considered significant. All statistical analyses were performed on a personal computer using the statistical software $\mathrm{R}$ version 2.15.0 [23]. This study

Table 1 Description of the seven stages of suture closure for MDCT evaluation

\begin{tabular}{ll} 
Stage & \\
\hline 0 & Completely open \\
1 & Inner bone cortex beginning to close $(<10 \%)$ \\
2 & Inner bone cortex closed \\
3 & Less than half closed \\
4 & More than half closed \\
5 & Visible relic of the ossification process \\
6 & Complete closure without relic \\
\hline
\end{tabular}

protocol was approved by the Ethical Review Board of the Graduate School of Medicine, Chiba University, and did not require approval of the kin.

\section{Results}

Pearson product-moment correlation coefficients between the age and the calculated representative scores were 0.79 with the "Average method," 0.77 with the "Frequency method," and 0.78 using the "Maximum method." Statistical significance tests of these correlation coefficients across the three methods indicated that the "Average method" had a better correlation than the "Frequency method" $(P=0.046)$, but no significant difference was observed between the "Frequency method" and "Maximum method" $(P=0.73)$ or between the "Maximum method" and "Average method" $(P=0.66)$. Accordingly, the mean "Average method" score was used as the representative value of sagittal suture closure, referred to as score A in further analyses. The intra- and inter-assessor difference in score A was tested with the Wilcoxon test, and there was no statistically significant difference in either the intra- (mean difference of $0.075 ; P=0.61$ ) and inter-assessor (mean difference of $0.038 ; P=0.65$ ) scoring.

To estimate age at death from score A, we performed regression analysis with age as a dependent variable and mean score as an explanatory variable. Table 2 and Fig. 3 show the coefficients of the formula with estimated $95 \%$ prediction intervals of \pm 31.4 years for all cases, \pm 33.6 years for men, and \pm 29.6 years for women. Minimum and maximum ages for each score A divided into 12 classes (classification score intervals of 0.5 ) are listed separately for males, females, and the entire sample in Table 3.

The presence of a gender difference in the degree of closure of the sagittal suture was also investigated using these results. Firstly, analysis of variance was performed to compare variance and indicated no significant difference between males and females $(P=0.013)$. Representative values of age at death between males and females were compared with the Mann-Whitney $U$ test, because age at death was not normally distributed, and this was found to be equivalent $(P=0.16)$. On the basis of their equivalent variance and representative age values, men and women were considered to represent the same population, and the influence of gender on the closure of sutures could be verified. Score A was not normally distributed, and central tendency demonstrated a median score A of 3.46 and inter-quartile range of 1.23-4.29 in men and a median score A of 4.38 and inter-quartile range of 2.14-5.05 in women. Mann-Whitney $U$ test indicated a statistically significant difference in the progression of closure degree of sutures between men and women $(P<0.01)$. As shown in Table 2, the coefficient of determination, which is interpreted as the goodness of fit of a 
Table 2 Regression analysis of score $\mathrm{A}$ and age at death

\begin{tabular}{llllll}
\hline & Number & Regression formula & $R^{2}$ & $P$ value & $95 \%$ prediction range (years) \\
\hline Total & 126 & $y=10.71 x+21.58$ & 0.63 & $<0.001$ & \pm 31.42 \\
Males & 65 & $y=9.12 x+27.01$ & 0.50 & $<0.001$ & \pm 33.55 \\
Females & 61 & $y=12.19 x+15.61$ & 0.74 & $<0.001$ & \pm 29.56 \\
\hline
\end{tabular}

regression, was higher for women $\left(R^{2}=0.74\right)$ than for men $\left(R^{2}=0.50\right)$. In order to investigate this gender difference, the correlation coefficient between age at death and score A was calculated by gender. Pearson product-moment correlation coefficients were 0.71 for men and 0.85 for women. Equivalence test of correlation coefficients between the genders indicated that this difference is statistically significant $(P=0.025)$.

\section{Discussion}

Our results revealed that evaluation of the degree of closure of the sagittal suture on cross-sectional MDCT images was positively correlated with age at death. This finding is consistent with the results reported by Harth et al. where flat-panel CT was used, but it differs from the conclusions of Obert et al. who reported that suture closure is not simply correlated to the aging process and is not useful in age estimation $[8,24]$. The software-based evaluation method of Obert et al. was considered accurate; however, the investigators employed an all-ornothing approach to judging the presence of suture closure. In contrast, similar to Harth et al., we evaluated closure degree by a multistep approach accounting for a difference in closure degree between the outer and inner table of the skull. This methodological difference is suspected to underlie the different outcomes. In order to put cross-sectional images to practical use in age estimation, visual examination using a multistep approach appears to be a minimal requirement.

The positive correlation seen here suggests that MDCT can be used to evaluate the degree of suture ossification and is, therefore, a potentially useful tool for age estimation. However, regression analysis shown in Table 2 revealed a very wide prediction interval of approximately 60 years, which is not superior to conventional methods such as flat-panel $\mathrm{CT}$ and naked-eye surface evaluation, which Schmitt and Tamáska reported as being 64 years for outer sutures and 50 years for inner sutures [16,21]. At this point, our method does not appear to advance age estimation accuracy, but our work demonstrates that this method can be easily used in departments where PMCT scanning is routinely performed. Furthermore, evaluation can be performed promptly after scanning without the removal of soft tissue. If used in conjunction with other methods, age estimation by sagittal suture evaluation on PMCT can be useful, especially in cases in which the skull is the sole remain. In these cases, teeth, being a hard tissue, also tend to be
Table 3 Minimum and maximum age at death for 12 intervals of score A

\begin{tabular}{|c|c|c|c|c|}
\hline \multirow[t]{2}{*}{ Score A } & & \multirow[t]{2}{*}{ Number } & \multicolumn{2}{|c|}{ Age at death (years) } \\
\hline & & & Minimum & Maximum \\
\hline \multirow[t]{12}{*}{ Total } & $0-0.5^{\mathrm{a}}$ & 20 & 0.08 & 59 \\
\hline & $0.5-1^{\mathrm{b}}$ & 8 & 19 & 48 \\
\hline & $1-1.5^{\mathrm{b}}$ & 4 & 21 & 72 \\
\hline & $1.5-2^{\mathrm{b}}$ & 3 & 43 & 59 \\
\hline & $2-2.5^{\mathrm{b}}$ & 7 & 26 & 68 \\
\hline & $2.5-3^{\mathrm{b}}$ & 1 & 68 & 68 \\
\hline & $3-3.5^{\mathrm{b}}$ & 12 & 24 & 76 \\
\hline & $3.5-4^{\mathrm{b}}$ & 11 & 37 & 92 \\
\hline & $4-4.5^{\mathrm{b}}$ & 19 & 40 & 93 \\
\hline & $4.5-5^{\mathrm{b}}$ & 19 & 43 & 93 \\
\hline & $5-5.5^{\mathrm{b}}$ & 13 & 62 & 91 \\
\hline & $5.5-6^{\mathrm{b}}$ & 8 & 48 & 96 \\
\hline \multirow[t]{12}{*}{ Males } & $0-0.5^{\mathrm{a}}$ & 10 & 0.666667 & 59 \\
\hline & $0.5-1^{\mathrm{b}}$ & 4 & 27 & 48 \\
\hline & $1-1.5^{\mathrm{b}}$ & 4 & 21 & 72 \\
\hline & $1.5-2^{\mathrm{b}}$ & 2 & 48 & 59 \\
\hline & $2-2.5^{\mathrm{b}}$ & 5 & 27 & 68 \\
\hline & $2.5-3^{b}$ & 1 & 68 & 68 \\
\hline & $3-3.5^{\mathrm{b}}$ & 7 & 43 & 76 \\
\hline & $3.5-4^{b}$ & 8 & 52 & 92 \\
\hline & $4-4.5^{\mathrm{b}}$ & 10 & 40 & 87 \\
\hline & $4.5-5^{\mathrm{b}}$ & 8 & 43 & 89 \\
\hline & $5-5.5^{\mathrm{b}}$ & 2 & 63 & 75 \\
\hline & $5.5-6^{\mathrm{b}}$ & 3 & 49 & 92 \\
\hline \multirow[t]{12}{*}{ Females } & $0-0.5^{\mathrm{a}}$ & 10 & 0.08 & 31 \\
\hline & $0.5-1^{b}$ & 4 & 19 & 48 \\
\hline & $1-1.5^{\mathrm{b}}$ & 0 & NA & NA \\
\hline & $1.5-2^{\mathrm{b}}$ & 1 & 43 & 43 \\
\hline & $2-2.5^{\mathrm{b}}$ & 2 & 26 & 36 \\
\hline & $2.5-3^{b}$ & 0 & NA & NA \\
\hline & $3-3.5^{\mathrm{b}}$ & 5 & 24 & 69 \\
\hline & $3.5-4^{b}$ & 3 & 37 & 60 \\
\hline & $4-4.5^{b}$ & 9 & 45 & 93 \\
\hline & $4.5-5^{\mathrm{b}}$ & 11 & 64 & 93 \\
\hline & $5-5.5^{\mathrm{b}}$ & 11 & 62 & 91 \\
\hline & $5.5-6^{\mathrm{b}}$ & 5 & 48 & 96 \\
\hline
\end{tabular}

${ }^{\mathrm{a}}$ Greater than or equal to 0 and less than or equal to 0.5

${ }^{\mathrm{b}}$ Greater than the left number and less than or equal to the right number 
relatively well preserved. Especially in young individuals, age estimation from dentition, using radiographic evaluation of root development and mineralization and macroscopic observation of tooth emergence patterns, is very accurate, and there are many dental standards available in the literature. However, after the permanent dentition is established, typically at around 14years of age, age estimation becomes increasingly difficult, and assessment of the mineralization of the third molars is the method of choice [26]. A study using this method on German individuals aged 15-40 years reported a standard error in age estimation of 5.3-5.7 years [27]. However, the use of this method in adults, particularly after middle age, is still controversial [28]. Accuracy of age estimation in adults can also be compounded by the high rate of congenital absence of the third molar, which in the modern Japanese population is about $30 \%$ [29]. Thus, age estimation in adults is typically based on the highly variable degeneration of bones although this is also reported to become inaccurate with increasing age $[10,28]$. As shown in Table 3, the age range of each score A class interval suggests possible upper and lower limits for accurate age determination with this method, which may be useful in some forensic investigation situations. For example, if the subject is in his or her 30 s or younger, a score of 4 suggests a high possibility of a negative match, and if the subject is known to be a woman of 50 years or older, a score lower than 0.5 also suggests a high possibility of a negative match. In this study, age estimation with regression formula was considered impractical because of the wide prediction interval as shown in Table 2 . However, as described above, assessment of the closure process by scoring of cross-section images can be used for age estimation in middle-aged and older subjects although age estimation in this age group remains challenging.

Some studies have confirmed gender differences in the ossification of cranial sutures, while others have refuted it $[15,16,18,19,25]$. In this study, analysis of age estimations based on the "Average method" scoring suggests that suture closure occurs more rapidly in women. Closure of sutures progresses by ossification, and bone metabolism is regulated by osteoblast-osteoclast cooperation. Estrogen is well known to affect bone metabolism, as is clear from the occurrence of postmenopausal osteoporosis, and thus, the observation that gender influences the degree of closure of sutures is in line with this evidence. In this study, there was also evidence of gender difference in the correlation between degree of closure and age, with this being stronger in women than in men. As shown in Fig. 3, multiple male subjects aged 60 or over showed relatively low score A values (i.e., $\leq 3$ ); however, this effect of age on correlation was not seen among female subjects. While the origin of these gender differences is not clear, the possible role of estrogen cannot be disregarded.

Of the 125 subjects, only two relatively young women, aged 48 and 55 years, had a score A of 6 in all four segments; interestingly, there was no score A of 6 among the subjects over
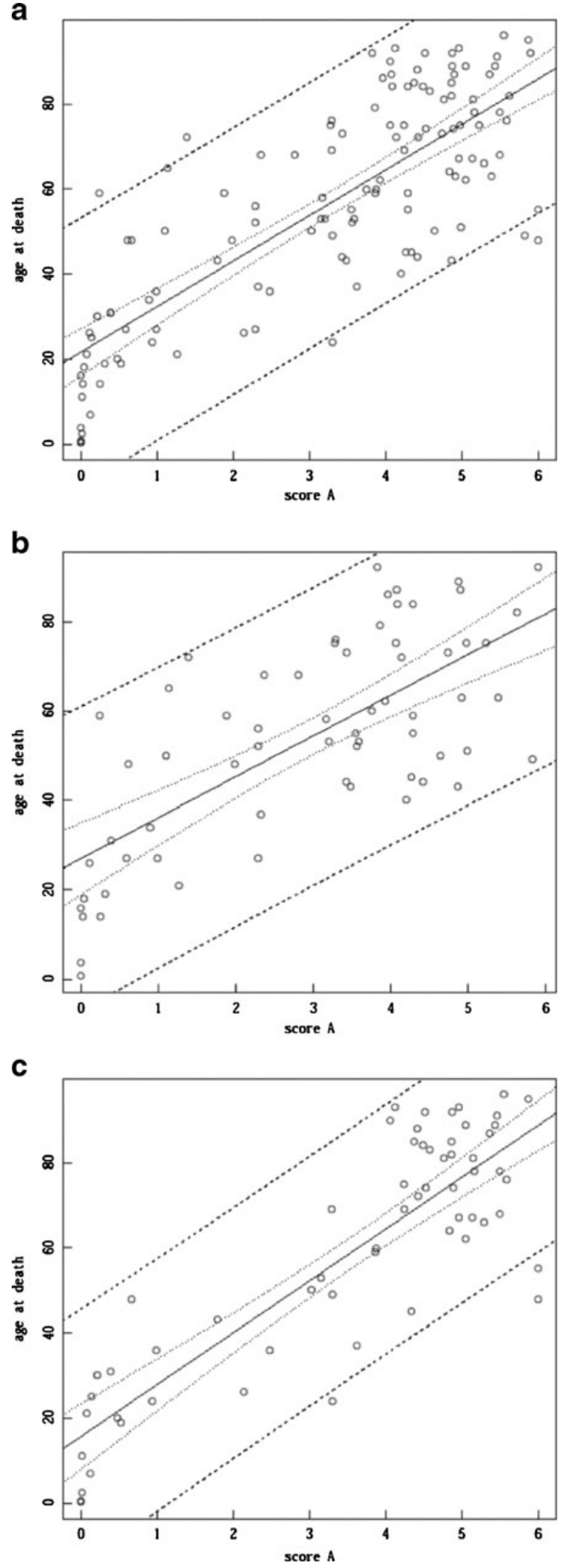

Fig. 3 Scatter plot of score A vs. age at death. a All cases, b males, c females. The solid line is the regression line, the fine dashed lines show the $95 \%$ confidence interval, and the coarse dashed lines show the $95 \%$ prediction range 
the age of 90 , many of whom demonstrated relics of the ossification process in at least one segment. These two subjects did not present any pathological abnormality of the skull such as deformed cranial bones seen in craniosynostosis patients. Not only the sagittal suture but also the coronal and lambdoid sutures were mainly closed in these subjects. Sahni et al. reported complete closure of both the ecto- and endocranial sagittal suture on macroscopic assessment; however, to the best of our knowledge, no reports on the early closure of the cranial suture without morphological aberration have been published [20]. Early closure of the cranial suture without morphological anomaly or other symptoms such as syndactylism was seen to be of no diagnostic value, which could explain its not having been reported previously. Some conditions such as hyperthyroidism, thalassemia, and hematologic and metabolic disorders are known to be associated with early suture fusion termed secondary craniosynostosis [30]. However, postmortem diagnosis of such diseases has limitations, and setting exclusion criteria to identify subjects with secondary craniosynostosis were not possible in the present study. Complete closure without relic over the whole suture represents a potential pitfall in suture assessment; therefore, in such cases, age estimation from cross-sectional images of sutures should be avoided.

Our study has some limitations. It may have failed to detect true differences such as the statistical significance of correlation coefficient across the three methods owing to small sample size. Additionally, all subjects were Mongoloid, and other races were not considered.

In conclusion, age estimation by the assessment of crosssectional MDCT images of the sagittal suture was possible, and a positive correlation between age and degree of closure was observed. Although this method is not superior to traditional macroscopic or flat-panel CT assessment with respect to prediction interval, our work shows that in conjunction with other methods, MDCT is a potentially useful tool for age estimation, particularly in adult women and in cases where a skull is the sole remain.

Conflict of interest The authors declare that they have no conflict of interest.

Ethical standards This experiment complies with the current laws of the country in which it was performed.

Open Access This article is distributed under the terms of the Creative Commons Attribution License which permits any use, distribution, and reproduction in any medium, provided the original author(s) and the source are credited.

\section{References}

1. Wade A, Nelson A, Garvin G, Holdsworth DW (2011) Preliminary radiological assessment of age-related change in the trabecular structure of the human os pubis. J Forensic Sci 56:312-319
2. Schulze D, Rother U, Fuhrmann A, Richel S, Faulmann G, Heiland M (2006) Correlation of age and ossification of the medial clavicular epiphysis using computed tomography. Forensic Sci Int 158:184-189

3. Aboshi H, Takahashi T, Komuro T (2010) Age estimation using microfocus X-ray computed tomography of lower premolars. Forensic Sci Int 200:35-40

4. Bassed RB, Briggs C, Drummer OH (2010) Analysis of time of closure of the spheno-occipital synchondrosis using computed tomography. Forensic Sci Int 200:161-164

5. Moskovitch G, Dedouit F, Braga J, Rougé D, Rousseau H, Telmon N (2010) Multislice computed tomography of the first rib: a useful technique for bone age assessment. J Forensic Sci $55: 865-870$

6. Dedouit F, Bindel S, Gainza D, Blanc A, Joffre F, Rougé D, Telmon N (2008) Application of the Iscan method to two- and threedimensional imaging of the sternal end of the right fourth rib. J Forensic Sci 53:288-295

7. Dang-Tran KD, Dedouit F, Joffre F, Rougé D, Rousseau H, Telmon N (2010) Thyroid cartilage ossification and multislice computed tomography examination: a useful tool for age assessment? J Forensic Sci 55:677-683

8. Harth S, Obert M, Ramsthaler F, Reuß C, Traupe H, Verhoff MA (2009) Estimating age by assessing the ossification degree of cranial sutures with the aid of flat-panel-CT. Legal Med 11:S186-S189

9. Harth S, Obert M, Ramsthaler F, Reuß C, Traupe H, Verhoff MA (2010) Ossification degrees of cranial sutures determined with flatpanel computed tomography: narrowing the age estimate with extrema. J Forensic Sci 55:690-694

10. Rissech C, Wilson J, Winburn AP, Turbón D, Steadman D (2012) A comparison of three established age estimation methods on an adult Spanish sample. Int J Legal Med 126:145-155

11. Schmidt S, Schmeling A, Zwiesigk P, Pfeiffer H, Schulz R (2011) Sonographic evaluation of apophyseal ossification of the iliac crest in forensic age diagnostics in living individuals. Int $\mathrm{J}$ Legal Med 125:271-276

12. Tisè M, Mazzarini L, Fabrizzi G, Ferrante L, Giorgetti R, Tagliabracci A (2011) Applicability of Greulich and Pyle method for age assessment in forensic practice on an Italian sample. Int J Legal Med 125:411-416

13. Sabancıoğulları V, Koşar Mİ, Salk I, Erdil FH, Oztoprak I, Cimen M (2012) Diploe thickness and cranial dimensions in males and females in mid-Anatolian population: an MRI study. Forensic Sci Int 219:289, e1-289.e7

14. Gy A, Nemeskéri J (1970) History of human life span and mortality. Akadémiai Kiadó, Budapest

15. Meindl RS, Lovejoy CO (1985) Ectocranial suture closure: a revised method for the determination of skeletal age at death based on the lateral-anterior sutures. Am J Phys Anthropol 68:57-66

16. Perizonius WRK (1984) Closing and non-closing sutures in 256 crania of known age and sex from Amsterdam (AD 1883-1909). J Hum Evol 13:201-216

17. Nawrocki SP (1998) Regression formulae for estimating age at death from cranial suture closure. In: Reichs KJ (ed) Forensic osteology: advances in the identification of human remains. Charles C. Thomas, Springfield, pp 276-292

18. Key CA, Aiello LC, Molleson T (1994) Cranial suture closure and its implications for age estimation. Int J Osteoarchaeol 4:193-207

19. Hershkovitz I, Latimer B, Dutour O, Jellema LM, Wish-Baratz S, Rothschild C, Rothschild BM (1997) Why do we fail in aging the skull from the sagittal suture? Am J Phys Anthropol 103:393-399

20. Sahni D, Jit I, Neelam S (2005) Time of closure of cranial sutures in northwest Indian adults. Forensic Sci Int 148:199-205

21. Cohen J, Cohen P (1983) Applied multiple regression/correlation analysis for the behavioral sciences, 2nd edn. Lawrence Erlbaum Associates, New Jersey, p57 
22. Steiger JH (1980) Tests for comparing elements of a correlation matrix. Psychol Bull 87:245-251

23. R Development Core Team (2012) R: a language and environment for statistical computing. R Foundation for Statistical Computing, Vienna, Austria. ISBN 3-900051-07-0, URL http://www.R-project.org/ Accessed 18 June 2012

24. Obert M, Schulte-Geers C, Schilling RL, Harth S, Kläver M, Traupe H, Ramsthaler F, Verhoff MA (2010) High-resolution flat-panel volumetric CT images show no correlation between human age and sagittal suture obliteration-independent of sex. Forensic Sci Int 200:180, e1

25. Schmitt HP, Tamáska L (1970) Investigation of cranial suture closure with special reference to the estimation of individual age. Z Rechtsmed 67:230-248
26. Thevissen PW, Galiti D, Willems G (2012) Human dental age estimation combining third molar(s) development and tooth morphological age predictors. Int J Legal Med 126:883-887

27. Olze A, Hertel J, Schulz R, Wierer T, Schmeling A (2012) Radiographic evaluation of Gustafson's criteria for the purpose of forensic age diagnostics. Int J Legal Med 126:615-621

28. Franklin D (2010) Forensic age estimation in human skeletal remains: current concepts and future directions. Leg Med (Tokyo) 12:1-7

29. Yamada H, Kondo S, Hanamura H (1993) The relationship of congenitally missing third molar to other missing teeth in human dentition. Jpn J Oral Biol 35:197-204

30. Ursitti F, Fadda T, Papetti L, Pagnoni M, Nicita F, Iannetti G, Spalice A (2011) Evaluation and management of nonsyndromic craniosynostosis. Acta Paediatr 100:1185-1194 